\title{
Colagem de barbotina de aluminas submicrométricas comerciais
}

\section{(Slip casting of commercial aluminas with fine powder)}

\author{
J. Catafesta ${ }^{1}$, R. Andreola ${ }^{1}$ C. A. Perottoni ${ }^{2,4}$, J. E. Zorzi ${ }^{3,4}$ \\ ${ }^{1} P G M A T,{ }^{2} C C E T / D E F Q,{ }^{3} C C E T / D E N Q$, Universidade de Caxias do Sul \\ R. Francisco Getúlio Vargas, 1130, Cidade Universitária, Caxias do Sul, RS 95070-560 \\ ${ }^{4}$ Instituto de Física, Universidade Federal do Rio Grande do Sul, Porto Alegre, RS 91501-970 \\ jezorzi@ucs.br
}

\begin{abstract}
Resumo
O processo de moldagem de peças cerâmicas conhecido como colagem de barbotina é um processo antigo, mas que ainda é muito utilizado devido ao seu baixo custo, simplicidade e pelas excelentes propriedades mecânicas das peças sinterizadas. Boas propriedades mecânicas são obtidas quando o pó de partida tem tamanho de partícula submicrométrico. Neste trabalho foram moldadas peças cerâmicas por colagem de barbotina com três aluminas comerciais com partículas submicrométricas e, após a sinterização, foram medidas algumas de suas propriedades, como dureza, densidade e resistência ao desgaste. As barbotinas não flocularam e foram obtidas peças de excelente qualidade, sendo que as peças moldadas com a alumina de menor tamanho de partícula apresentaram a melhor dureza, elevada densidade (chegando a 99\% da densidade teórica) e o menor coeficiente de desgaste. Peças de alumina de alta qualidade podem ser usadas em inúmeras aplicações, desde a indústria têxtil até em aplicações biomédicas.
\end{abstract}

Palavras-chave: alumina, colagem, sinterização, propriedades.

\begin{abstract}
The molding of ceramics parts by slip casting is an ancient process, but it is still very used because of low cost, simplicity and excellent mechanical properties of sintered parts. Better mechanical properties are obtained when the initial powder have small average particle size. In this work, ceramic parts were molded by slip casting of three commercial submicrometer-sized alumina powders. After sintering, some properties of these ceramic parts were measured, including hardness, density and wear coefficient. The slips do not flocullate, resulting in ceramic parts with excellent quality. Ceramic parts made with the alumina powder of smaller particle size exhibited high hardness, high density (99\% TD) and lower wear coefficient. High quality alumina parts such as that produced in this work can be used in many applications, from textile industry to biological applications.
\end{abstract}

Keywords: alumina, slip casting, sintering, properties.

\section{INTRODUÇÃO}

A colagem de barbotina é um processo muito antigo e largamente empregado na produção de peças cerâmicas devido à sua relativa simplicidade e baixo custo de investimento. É normalmente descrito como a consolidação de partículas cerâmicas de uma suspensão coloidal, através da remoção da parte líquida, por um molde absorvente [1]. Em outras palavras, na suspensão (barbotina) a parte líquida, geralmente água, é retirada por meio de um molde poroso, normalmente gesso. Quando a água é absorvida pelo molde, ocorre a aproximação das partículas de pó, em suspensão na água, na superfície do molde. Após a formação da parede com a espessura desejada, o eventual excedente de barbotina pode ser drenado, deixando-se o que resta de água na parede formada ser absorvido pelo molde, quando então temos a peça a verde [2]. Entre as vantagens deste método temos a obtenção de formas relativamente complexas, com paredes finas e uniformes, economia em pequenas produções e moldes mais baratos.

Apesar da técnica de colagem de barbotina ser antiga, é necessário o desenvolvimento de uma formulação (pó cerâmico + aditivos de moldagem) adequada, de acordo com as características de cada pó cerâmico. Se a barbotina não estiver bem dispersa, as propriedades das peças podem ficar aquém do desejado, gerando peças defeituosas, com paredes irregulares. Estas peças defeituosas terão suas propriedades mecânicas ou estruturais prejudicadas, após a sinterização, por defeitos ocorridos no processo de conformação. Então, o objetivo deste trabalho foi desenvolver barbotinas com diferentes pós de alumina submicrométrica e comparar algumas de suas principais propriedades, como dureza, densidade e resistência ao desgaste. A partir de pós muito finos podemos obter peças cerâmicas com porosidade menor e, consequentemente, maior densidade e melhores propriedades mecânicas. Peças com alta qualidade possuem aplicações nas mais diversas áreas, desde guia-fios da indústria têxtil e bicos de jateamento abrasivo até aplicações biomédicas [3].

\section{Princípios físicos}

$\mathrm{O}$ processo de colagem de barbotina se assemelha à filtração. Em ambos os casos existem diferenciais de pressão 
que provocam a difusão da água através dos sólidos. $\mathrm{O}$ diferencial de pressão na colagem se deve às ações capilares que transportam a água através do gesso [1].

A taxa de crescimento da espessura da camada depositada na parede do molde de gesso diminui com o inverso da espessura da parede formada [4]:

$$
\frac{d L_{1}}{d t}=\frac{(-\Delta P)}{2 C L_{1}}
$$

Após integração,

$$
t=\frac{C L_{1}^{2}}{(-\Delta P)}
$$

onde $t$ é o tempo necessário para a formação de uma camada depositada de espessura $L_{l}$ e $\Delta P$ é a queda de pressão através da parede. A constante $C$ inclui a viscosidade do líquido, a quantidade de líquido na suspensão, o fator de empacotamento das partículas e o coeficiente de permeabilidade. Estas equações supõem um depósito nãocompressível e, como isso não é completamente verdadeiro, a espessura do depósito irá aumentar mais lentamente do que o previsto [5]. A velocidade de deposição varia com a quantidade de água na suspensão: quanto mais água, mais vagaroso o processo, pois a água deve ser filtrada através do depósito formado. Altos valores de viscosidade são marcados por altas velocidades de deposição. Velocidades de deposição mais lentas produzem melhor empacotamento das partículas e melhor densidade a verde [6]. Deste modo, uma barbotina deve ter baixa viscosidade para que ocorra uma melhor formação de camada e para evitar o aparecimento de bolhas de ar. Portanto, uma boa dispersão é necessária também para assegurar a máxima fluidez com um mínimo de água.

Através da colagem de barbotina, se pode conseguir corpos a verde com alta densidade, devido às grandes forças capilares envolvidas que atuam de modo a formar uma parede compacta de partículas sobrepostas, principalmente em se tratando de um pó formado por partículas pequenas.

\section{Aditivos}

O meio mais usado na colagem é a suspensão aquosa, onde a aglomeração e a decantação são evitadas com o uso de dispersantes ou defloculantes [7].

$\mathrm{O} \mathrm{pH}$ no qual o potencial zeta $(\zeta)$ é nulo é chamado de ponto isoelétrico. Neste $\mathrm{pH}$ a repulsão entre as partículas é mínima e a suspensão flocula resultando em uma suspensão muito viscosa (o ponto isoelétrico da $\alpha-\mathrm{Al}_{2} \mathrm{O}_{3}$ varia entre 8 e 9) [8]. Suspensões muito viscosas formam peças com paredes irregulares [1,9], mas se o $\mathrm{pH}$ da suspensão estiver suficientemente distante do ponto isoelétrico a mesma poderá se manter dispersa sem o uso de defloculante.

Para que a dispersão ocorra, é necessário que seja alcançada uma condição de equilíbrio entre as forças de atração e repulsão entre as partículas e a força de gravidade.
A função do dispersante é evitar aglomerações de partículas, o que pode ser conseguido tornando-as eletricamente carregadas. Para isso modifica-se o $\mathrm{pH}$ da suspensão adicionando-se uma base $\left(\mathrm{OH}^{-}\right)$ou um ácido $\left(\mathrm{H}^{+}\right)$. Quando as cargas das partículas são idênticas, há uma repulsão eletrostática entre elas. Se estas forças são suficientes para superar a atração de van der Waals, o sistema ficará bem disperso. Este estado de dispersão não é único, pois existem vários graus de dispersão, dependendo da magnitude das forças repulsivas.

As suspensões devem possuir valores de pH intermediários, de modo a evitar o desgaste do molde de gesso por corrosão. Apesar disso, em suspensões dispersas sem uso de defloculante, costuma-se usar valores extremos de $\mathrm{pH}$ para se obter uma suspensão estável por longo período [6].

Uma suspensão bem dispersa mostra um comportamento próximo ao newtoniano, em contraste com a floculada, que tem um comportamento pseudoplástico [6]. As características reológicas da suspensão têm grande influência sobre o processo de formação do compacto a verde e seus eventuais defeitos. A reologia da suspensão depende, entre outras coisas, dos aditivos e impurezas presentes, da distribuição de tamanho de partículas, da área superficial e de modificações coloidais [6].

Outros fatores que afetam a moldagem, além da viscosidade, $\mathrm{pH}$ e dispersante são a concentração de sólidos e as condições de moldagem. Controlando estes fatores se obtém os melhores compactos a verde [7].

Existe uma quantidade ideal de dispersante. Dispersante em excesso pode se comportar como aglutinante e com isso as peças aderem ao molde e não se consegue extraí-las. Pode-se adotar um valor um pouco abaixo do ideal por razões de cinética de formação de parede [10]. Por exemplo, uma suspensão totalmente defloculada tende a formar uma parede com menor permeabilidade do que uma parcialmente dispersa [7].

$\mathrm{O}$ ácido cítrico vem sendo usado como dispersante em suspensões de alumina, principalmente porque forma um ânion carboxilato na superfície, provocando mudanças na carga superficial e, consequentemente, a repulsão entre as partículas [11, 12].

Outro aditivo que se torna necessário para a conformação de peças cerâmicas por colagem de barbotina é o ligante. $\mathrm{O}$ álcool polivinílico (PVA) é um exemplo de aditivo que pode ser usado como ligante [12]. O propósito da adição do ligante é aumentar a resistência do corpo a verde, o suficiente para que ele possa ser manipulado e receber acabamento [13]. Mas a presença do ligante provoca aumento da viscosidade da barbotina, o que faz com que a concentração de sólidos deva ser diminuída, reduzindo assim a velocidade de formação de parede da peça a verde $[1,13]$.

\section{Moldes}

Os moldes utilizados na colagem de barbotina geralmente são de gesso de alta permeabilidade e pequeno tamanho de poro de modo a absorver a água da barbotina sem escoamento das partículas em suspensão para dentro da parede do molde. 
Normalmente o diâmetro dos poros dos moldes de gesso é em torno de $0,1 \mu \mathrm{m}$, o que é menor que o diâmetro médio de partículas usualmente empregado em suspensões. Deste modo não ocorre migração significativa das partículas para dentro dos capilares do molde.

A vantagem deste tipo de molde são o custo relativamente baixo, simplicidade no manuseio, porosidade controlada e boa exatidão dimensional. Estes moldes podem ser secados e reaproveitados.

\section{MATERIAIS E MÉTODOS}

\section{Matérias-primas}

Neste trabalho utilizaram-se como matérias-primas, três aluminas $\left(\mathrm{Al}_{2} \mathrm{O}_{3}\right)$ comercias: A-16SG, A-1000SG e CT3000SG, todas da empresa Alcoa S.A.. As aluminas foram utilizadas como recebidas e a composição e propriedades segundo o fabricante são as seguintes:

Alumina A-16SG: $\mathrm{Al}_{2} \mathrm{O}_{3}-99,82 \%, \mathrm{~N}_{2} \mathrm{O}-0,07 \%, \mathrm{SiO}_{2}$ $-0,03 \%, \mathrm{Fe}_{2} \mathrm{O}_{3}-0,02 \%, \mathrm{CaO}-0,02 \%$ e $\mathrm{MgO}-0,05 \%$. Área superficial $=8,9 \mathrm{~m}^{2} / \mathrm{g}$; porcentagem que passou pela peneira 325 mesh $=99,9 \%$; densidade a verde $(5000 \mathrm{psi})=2,18 \mathrm{~g} /$ $\mathrm{cm}^{3}$; densidade sinterizada $=3,90 \mathrm{~g} / \mathrm{cm}^{3}\left(1540{ }^{\circ} \mathrm{C}\right)$; retração na sinterização $=17,5 \%$; tamanho médio de partícula $=0,4 \mu \mathrm{m}$.

Alumina A-1000SG: $\mathrm{Al}_{2} \mathrm{O}_{3}-99,8 \%, \mathrm{~N}_{2} \mathrm{O}-0,07 \%, \mathrm{SiO}_{2}$ $-0,03 \%, \mathrm{Fe}_{2} \mathrm{O}_{3}-0,02 \%, \mathrm{CaO}-0,02 \%$ e $\mathrm{MgO}-0,04 \%$. Área superficial $=8,4 \mathrm{~m}^{2} / \mathrm{g}$; porcentagem que passou pela peneira 325 mesh $=99,9 \%$; densidade a verde $(5000 \mathrm{psi})=2,14 \mathrm{~g} /$ $\mathrm{cm}^{3}$; densidade sinterizada $=3,85 \mathrm{~g} / \mathrm{cm}^{3}\left(1540{ }^{\circ} \mathrm{C}\right)$; retração na sinterização $=17, \%$; tamanho médio de partícula $=0,4 \mu \mathrm{m}$.

Alumina CT-3000SG: $\mathrm{Al}_{2} \mathrm{O}_{3}-99,8 \%, \mathrm{~N}_{2} \mathrm{O}-0,08 \%, \mathrm{SiO}_{2}$ $-0,03 \%, \mathrm{Fe}_{2} \mathrm{O}_{3}-0,02 \%, \mathrm{CaO}-0,02 \%$ e $\mathrm{MgO}-0,07 \%$. Área superficial $=7,5 \mathrm{~m}^{2} / \mathrm{g}$; porcentagem que passou pela peneira 325 mesh $=99,9 \%$; densidade pressionada $(90 \mathrm{MPa})=2,25 \mathrm{~g} /$ $\mathrm{cm}^{3}$; densidade sinterizada $=3,90 \mathrm{~g} / \mathrm{cm}^{3}\left(1540{ }^{\circ} \mathrm{C}\right)$; retração na sinterização $=16,8 \%$; tamanho médio de partícula $=0,5 \mu \mathrm{m}$.

\section{Formulações}

Vários experimentos foram realizados na tentativa de estabelecer a melhor formulação para a barbotina A-16SG, pois para partículas de pequeno tamanho, freqüentemente encontram-se dificuldades na sua dispersão. Os experimentos iniciais foram realizados a partir da análise da literatura $[6,7,14]$ e foram sendo alterados até se obter a melhor formulação para este pó cerâmico. Uma vez obtida uma formulação estável e com baixa viscosidade, a mesma formulação foi aplicada à alumina A-1000SG, pois os dois pós possuem tamanho médio de partícula semelhantes e não foram necessárias alterações. A formulação utilizada foi a seguinte (em massa):

$69,4 \%$ de alumina;

$24,7 \%$ de água deionizada;

$0,8 \%$ de ácido cítrico (reagente analítico - Vetec 99,5\% diluído a $10 \%$ );

0,12\% de solução do defloculante IQA PAC-C (Indústrias
Químicas Arujá Ltda - solução 1:3);

$5 \%$ de ligante álcool polivinílico (PVA) Airvol 540 - alta densidade (diluído a $10 \%$ );

algumas gotas de antiespumante IQA E05 (Indústrias Químicas Arujá Ltda).

O pH das barbotinas das aluminas A-16SG e A-1000SG foi de aproximadamente 8,8 .

Estes materiais são misturados em moinho de bolas de alumina por $20 \mathrm{~h}$. Após a barbotina ser retirada do moinho, deixa-se em repouso para a eliminação das bolhas de ar que ainda restam e após, está pronta para ser vertida nos moldes de gesso previamente preparados e secos em estufa com circulação de ar.

Após a colagem, as peças secam dentro dos moldes à temperatura ambiente até a retirada da maior quantidade de água possível e, após a abertura dos moldes, as peças terminam de secar ao ar.

Em seguida, as peças são pré-sinterizadas a $1000{ }^{\circ} \mathrm{C} / 1 \mathrm{~h} \mathrm{e}$, após receberem acabamento, são sinterizadas ao ar, sobre uma superfície refratária recoberta com uma fina camada de pó de alumina A-1 (da Alcoa), com rampa de aquecimento de $1^{\circ} \mathrm{C} / \mathrm{min}$ até $1600^{\circ} \mathrm{C}$. A sinterização se processa, a esta temperatura por mais duas horas. Dependendo da aplicação, após a sinterização, as peças podem ser polidas com pastas diamantadas para melhorar o acabamento e diminuir o atrito superficial.

Com a formulação acima a barbotina de alumina CT3000SG ficou muito viscosa, sendo necessário desenvolver uma nova formulação para este pó:

$64,0 \%$ de alumina;

$29,0 \%$ de água deionizada;

$1,0 \%$ de ácido cítrico (reagente analítico - Vetec 99,5\% diluído a $10 \%$ );

1,0\% de Dispersal 130 (BetzDearborn Brasil Ltda);

5\% de ligante álcool polivinílico (PVA) Airvol 540 - alta densidade (diluído a $10 \%$ );

algumas gotas de antiespumante IQA E05 (Indústrias Químicas Arujá Ltda).

$\mathrm{O}$ pH desta suspensão foi de 8,6. A queima das peças de alumina CT-3000SG seguiu os mesmos passos descritos acima.

\section{Análises das peças sinterizadas}

Para a análise de algumas propriedades das peças produzidas durante este trabalho foram realizados ensaios de microdureza, densidade e resistência ao desgaste. Para as medidas de dureza e de desgaste, as amostras foram embutidas em resina e polidas com pastas diamantadas. Utilizou-se um microdurômetro Shimadzu (type $M$ ), equipado com uma ponteira de diamante Knoop, e as amostras foram ensaiadas com carga de $500 \mathrm{~g}$ por $15 \mathrm{~s}$. O coeficiente de desgaste foi estimado usando um Calowear Tester (modelo CSEM, Centre Suisse d'Electronique et de Microtechnique). Neste ensaio, uma esfera de aço, em contato com a superfície da amostra, gira acionada por um eixo. Sobre a esfera é gotejada uma suspensão abrasiva de $\mathrm{SiC}$, cujo tamanho médio de partícula situa-se em torno de $5 \mu \mathrm{m}$, que provocará o desgaste da amostra. O resultado será a formação de uma cratera, cujo diâmetro é 
medido por microscopia óptica. A partir deste diâmetro podese calcular o coeficiente de desgaste, que é definido como o volume de material arrancado por distância percorrida e pela força aplicada pela esfera $[15,16]$. Um esquema deste ensaio e um exemplo de cratera obtida podem ser vistos na Fig. 1. A densidade das amostras sinterizadas foi medida em balança analítica utilizando-se o método de Arquimedes.

As amostras polidas foram aquecidas a $1450{ }^{\circ} \mathrm{C}$ por 60 min para revelar o contorno de grão. Estas amostras foram observadas em microscópio eletrônico de varredura da marca Jeol JSM 6060.
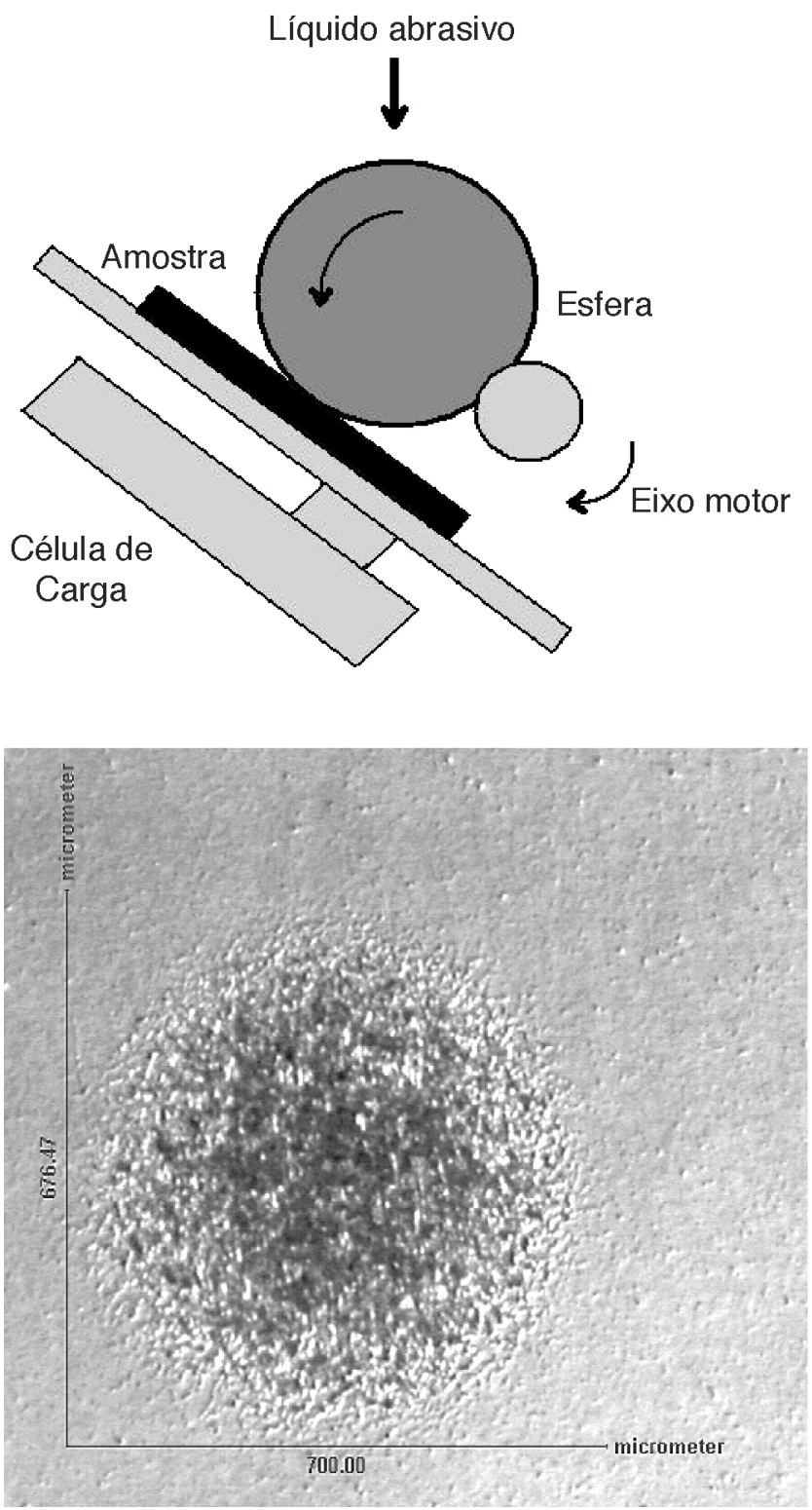

Figura 1: Esquema do ensaio de desgaste utilizando o equipamento Calowear (adaptado de [17]) e exemplo de cratera obtida por este método de ensaio.

[Figure 1: Schematic representation of the Calowear tester equipment (adapted from [17]) and an example of crater obtained by this method.]

\section{RESULTADOS E DISCUSSÃO}

As barbotinas não ficaram bem defloculadas somente com a adição do dispersante comercial. Para reduzir a viscosidade das suspensões foi necessário adicionar ácido cítrico. O mesmo pode ser dito em relação ao ligante PVA. $\mathrm{O}$ índice de perda de peças por quebra no manuseio foi muito elevado. Sem o PVA as peças não podem ser manuseadas e nem receber acabamento pré-queima, uma vez que não
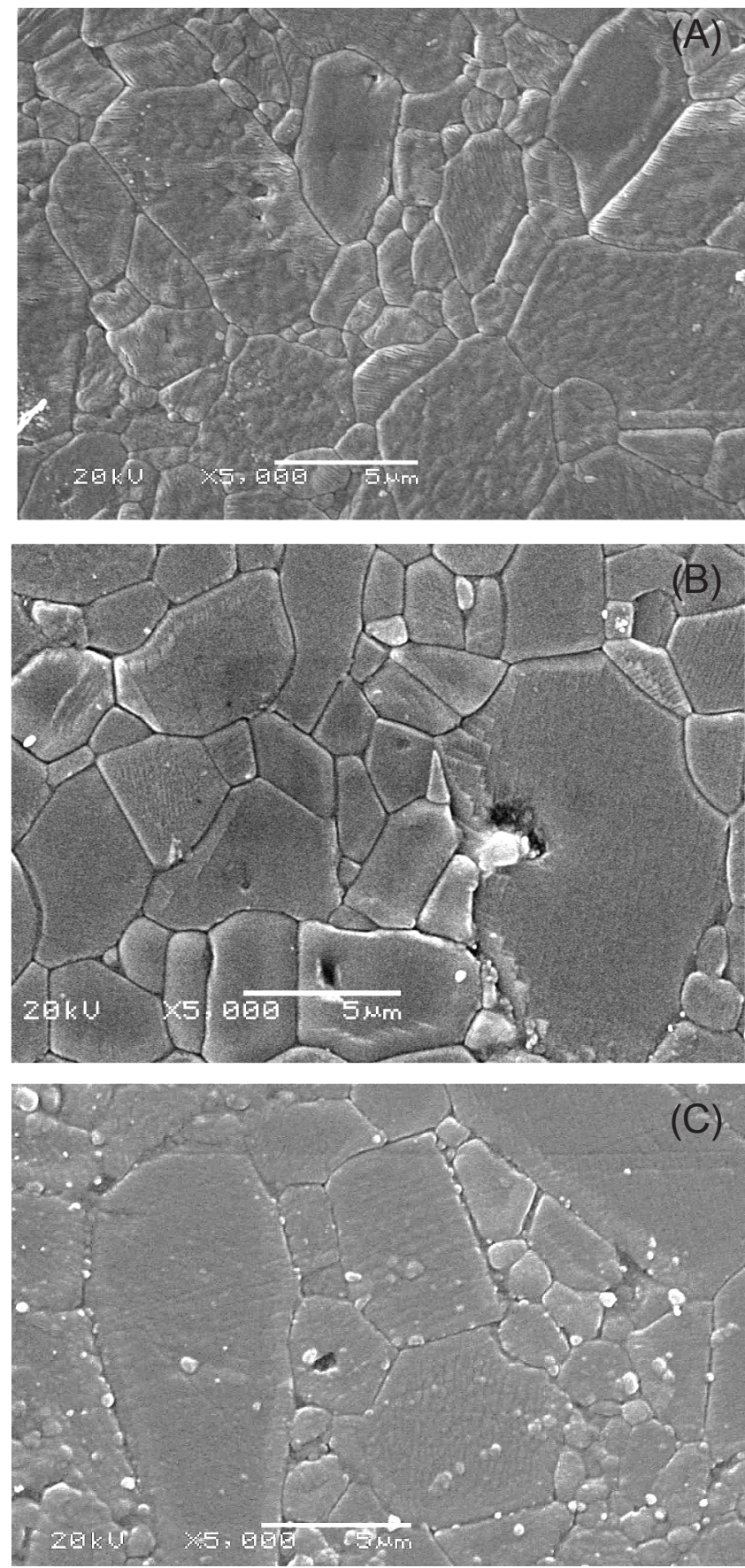

Figura 2: Micrografias obtidas por microscopia eletrônica de varredura (5000x): (a) A-16SG, (b) A-1000SG e (c) CT-3000SG. [Figure 2: SEM micrographs (5000x): (a) A-16SG, (b) A-1000SG and (c) CT-3000SG.] 
Tabela I - Resultados dos ensaios nas amostras das aluminas.

[Table I - Experimental results obtained with some alumina samples.]

\begin{tabular}{ccccc}
\hline Alumina & $\begin{array}{c}\text { Densidade } \\
\left(\mathrm{g} / \mathrm{cm}^{3}\right) \\
\text { teórica }\end{array}$ & $\begin{array}{c}\% \\
\text { densidade } \\
\left(\mathrm{kgf} / \mathrm{mm}^{2}\right)\end{array}$ & $\begin{array}{c}\text { Dureza } \\
\text { Knoop } \\
(500 \mathrm{~g})\end{array}$ & $\begin{array}{c}\text { Coeficiente } \\
\text { de Desgaste } \\
\left(\mathrm{x} 10^{-13} \mathrm{~m}^{2} \mathrm{~N}^{-1}\right)\end{array}$ \\
\hline A-16SG & $3,94 \pm 0,01$ & $99,0 \pm 0,3$ & $1677 \pm 26$ & $7,7 \pm 0,1$ \\
A-1000SG & $3,93 \pm 0,01$ & $98,7 \pm 0,3$ & $1628 \pm 27$ & $7,3 \pm 0,1$ \\
A-3000SG & $3,91 \pm 0,01$ & $98,2 \pm 0,3$ & $1580 \pm 26$ & $7,4 \pm 0,1$ \\
\hline
\end{tabular}

possuem suficiente resistência mecânica. Uma vez que a suspensão foi vertida nos moldes, o tempo necessário para a formação das peças é de 1 a $6 \mathrm{~h}$, dependendo da espessura de parede desejada. Como o $\mathrm{pH}$ é um pouco elevado, o número de peças que podem ser coladas por molde não é muito grande, sendo que a qualidade vai diminuindo com o uso.

A densidade das peças cerâmicas sinterizadas, variou entre $98,2 \%$ a $99 \%$ da densidade teórica da alumina (Tabela I), dependendo do pó cerâmico, da espessura e da geometria das peças. Depois de sinterizadas, o tamanho de partícula cresceu de uma ordem de magnitude em relação ao pó cerâmico (Fig. 2). Também foi observado que todas as aluminas ficaram bem sinterizadas, formando um corpo cerâmico de alta densidade e excelentes propriedades mecânicas, sendo que os melhores resultados das medidas de densidade foram obtidos com a alumina A-16SG (Tabela I), valores estes que foram comparados com a literatura [18].

Nos ensaios de microdureza Knoop, os melhores resultados obtidos também foram com a alumina A-16SG (Tabela I). Os resultados das medidas do coeficiente de desgaste, que também podem ser vistos na Tabela I, não sugerem uma diferença significativa entre as aluminas estudadas, e aparentemente não possuem relação direta com a densidade e a dureza obtidas.

Os resultados obtidos demonstram que é possível a produção de peças de alumina para os mais variados fins, com alta qualidade, pelo método de colagem de barbotina, a partir de diferentes pós submicrométricos, de modo a

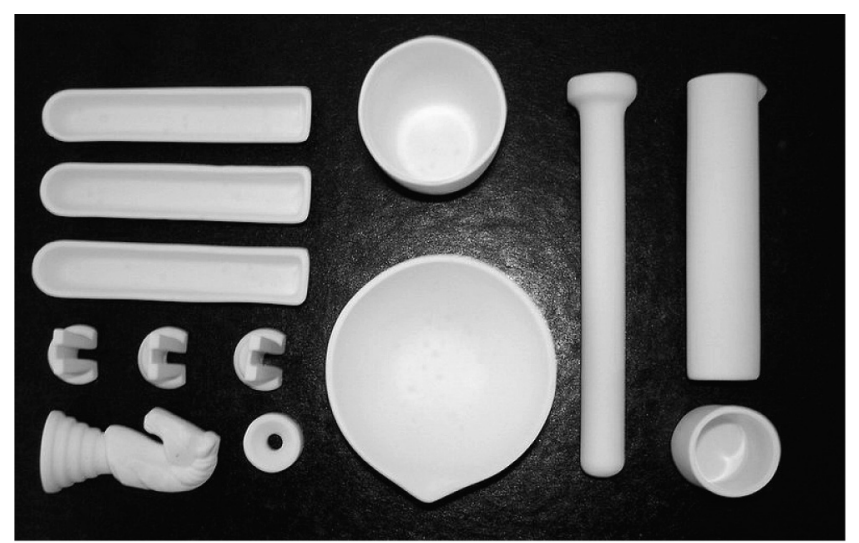

Figura 3: Peças de alumina submicrométrica, feitas por colagem de barbotina.

[Figure 3: Ceramic parts made with submicrometer-size alumina powder, molded by slip casting.] suprir uma parte da demanda do mercado brasileiro de peças cerâmicas de alta tecnologia. Com polimento, estas peças apresentam excelente acabamento superficial, podendo ser usadas nas áreas biomédica e têxtil. Como o coeficiente de desgaste é baixo, estas peças possuem boa durabilidade e podem ser usadas inclusive para bicos de jateamento. Algumas peças produzidas neste trabalho, já sinterizadas, podem ser vistas na Fig. 3.

\section{CONCLUSÕES}

O processo de colagem de barbotina é adequado para a produção de peças de aluminas submicrométricas, com formatos relativamente complexos, de modo a suprir uma parte da demanda do mercado brasileiro de peças cerâmicas de alta tecnologia. A formulação desenvolvida não floculou e apresentou boa fluidez, o que gerou peças homogêneas com elevada densidade, dureza e baixo coeficiente de desgaste.

Dos resultados obtidos, conclui-se também que as peças sinterizadas obtidas a partir de aluminas submicrométricas possuem excelentes propriedades e podem ser usadas para as mais diversas aplicações.

\section{AGRADECIMENTOS}

Este trabalho foi parcialmente financiado por FAPERGS, CAPES e CNPq. Os autores agradecem a Alcoa S.A. pelas amostras de alumina usadas neste trabalho.

\section{REFERÊNCIAS}

[1] J. S. Reed, Principles of Ceramics Processing, John Wiley \& Sons, Inc., NY (1995).

[2] J. E. Zorzi, Conformação de Cerâmicas de Alta Tecnologia com Formas Complexas. Dissertação de Mestrado, PPGEM - UFRGS (1996).

[3] S. F. Hulbert, The use of alumina and zirconia in surgical implants. In: An Introduction to Bioceramics 1. World Sci. L. L. Hench and J. Wilson Ed., Singapure (1993) 25.

[4] L. H. Van Vlack, Propriedades dos Materiais Cerâmicos. Ed. Blücher Ltda, SP (1973).

[5] G. G. Brown, A. S. Foust, D. L. V. Katz, R. Schneidewind, R. R. White, W. P. Wood, G. M. Brown, L. E. Brownell, J. J. Martin, G. B. Willians, J. T. Banchero, J. L. York, Unit Operations, John Wiley \& Sons, NY (1950). [6] U. Mattos, S. L. Vendrasco, R. T. T. Noronha, V. C. Pandolfelli, J. A. Rodrigues, R. P. Jesus, Anais do $36^{\circ}$ Congresso Brasileiro de Cerâmica, Caxambú, MG, 2 (1992) 816.

[7] J. M. Keller, R. R.Ulbrich, R. A. Haber, Am. Ceram. Soc. Bull. 76, 3 (1997) 87.

[8] J. A. Lewis, J. Am. Ceram. Soc. 83, 10 (2000) 2341.

[9] R. Herrmann, Slip Casting in Practice. Ceramics Monographs - Handbook of Ceramics. Suplement to Interceram. 38, 4 (1989) no 1.4.2.1.

[10] W. D. Kingery, Introduction to Ceramics. $2^{\text {nd }}$ Edition, John Wiley \& Sons, Inc., NY (1976). 
[11] P. C. Hidber, T. J.Graule, L. J. Gauckler, J. Am. Ceram. Soc. 79, 7 (1996) 1857.

[12] P. C. Hidber, T. J.Graule, L. J. Gauckler, J. Am. Ceram. Soc. 78, 7 (1995) 1775.

[13] W. B. Cavazzani, D. Riviére, Q. B. Corrêa, Anais do $13^{\circ}$ Congresso Brasileiro de Engenharia e Ciência dos Materiais, Curitiba, PR (1998) 3205.

[14] G. W. Phelps, Slip casting. Ceramics Monographs - Handbook of Ceramics. Verlag Schmid GmbH, Freiburg
(1982) $\mathrm{n}^{\circ}$ 1.4.2.

[15] K. L. Rutherford, I. M. Hutchings, Surf. Coat. Technol. 79 (1996) 231.

[16] M. G. Gee, A. Gant, I. Hutchings, R. Bethke, K. Schiffman, K. Van Acker, S. Poulat, Y. Gachon, J. von Stebut, Wear 255 (2003) 1.

[17] Disponível em: http://www.csm-instruments.com/ frames/princ_spher.htmlAcesso em: 07 de março de 2006.

[18] R. G. Munro, J. Am. Ceram. Soc. 80, 8 (1997) 1919. (Rec.29/03/2006, Rev. 29/06/2006, Ac. 04/08/2006) 